\title{
Derivative Pricing under the Possibility of Long Memory in the supOU Stochastic Volatility Model
}

\author{
Robert Stelzer and Jovana Zavišin
}

\begin{abstract}
We consider the supOU stochastic volatility model which is able to exhibit long-range dependence. For this model, we give conditions for the discounted stock price to be a martingale, calculate the characteristic function, give a strip where it is analytic, and discuss the use of Fourier pricing techniques. Finally, we present a concrete specification with polynomially decaying autocorrelations and calibrate it to observed market prices of plain vanilla options.
\end{abstract}

Keywords Calibration - Fourier pricing $\cdot$ Lévy basis $\cdot$ Long memory $\cdot$ Superposition of Ornstein-Uhlenbeck-type processes $\cdot$ Stochastic volatility

AMS Subject Classification 2010 Primary: 91G20, 60G51 · Secondary: 91B25

\section{Introduction}

The Ornstein-Uhlenbeck (OU)-type stochastic volatility (SV) model introduced in [3] is one of the most popular stochastic volatility models for prices of financial assets driven by a Lévy process (see, e.g., [11, 25]). It covers many of the stylized facts typically encountered in financial data (cf. [10, 14]). Over the years many variants have been introduced, for instance a variant with two sided jumps in [1] or a multivariate extension in [21].

The views expressed herein are those of the authors and do not necessarily reflect the views of the institutions mentioned below.

\footnotetext{
R. Stelzer $(\varangle)$

Institute of Mathematical Finance, Ulm University, Helmholtzstr. 18,

89081 Ulm, Germany

e-mail: robert.stelzer@uni-ulm.de

J. Zavišin

risklab GmbH/Allianz Global Investors, Seidlstr. 24-24a,

80335 Munich, Germany

e-mail: jovana.zavisin@ risklab.com

(C) The Author(s) 2015

K. Glau et al. (eds.), Innovations in Quantitative Risk Management,

Springer Proceedings in Mathematics \& Statistics 99,

DOI 10.1007/978-3-319-09114-3_5
} 
In this paper, we consider a variant of the model which additionally can cover the stylized fact of long-range dependence (or slower than exponentially decaying autocorrelations), the supOU stochastic volatility model. In this model, we specify the volatility as a superposition of Ornstein-Uhlenbeck (thus "supOU") processes, which have been introduced in [2]. Various features of this volatility model (in a multidimensional setting) have been considered in $[4,5,18,26]$.

Typically long-range dependence is obtained by using fractional Brownian motion or fractional Lévy processes as the driving noises, see, e.g., [6, 7] for a critical discussion of such models for financial markets. In such models one cannot have jumps, as fractional Lévy processes (cf. [16]) have continuous paths, and one is bound to have long memory. In our supOU model, one has a natural extension of the OU-type model that exhibits jumps and, depending on the parameters, can exhibit short or long memory. However, our model shares one disadvantage with fractional process based models, viz. that it is no longer Markovian. In this context, one should bear in mind that most Markov processes one employs to model volatilities are geometrically ergodic and thus cannot exhibit long memory, although there exists also Markov process with polynomial mixing coefficients and even long memory (see, e.g., [27]).

The focus of the present paper is on derivative pricing in and calibration of the univariate supOU SV model similar to the papers $[19,20]$ in the (multivariate) OUtype SV model. To this end, we first briefly review the model in Sect.2. In Sect.3, we give conditions on the parameters such that the discounted stock price process is a martingale which implies that under these conditions the model can be used to describe the risk neutral dynamics of a financial asset. Thereafter, we start Sect. 4 with a review of Fourier pricing. Then, we give the characteristic function of the log asset price in the supOU SV model and show conditions for the moment generating function to be sufficiently regular so that Fourier pricing is applicable. Finally, we present a concrete specification, the $\Gamma$-supOU SV model, in Sect. 5 and discuss its calibration to market data which we illustrate with a small example using options on the DAX. Finally, we discuss a subtle issue regarding how to employ the calibrated model to calculate prices of European options with a general maturity.

\section{A Review of the supOU Stochastic Volatility Model}

We briefly review the definition and the most important known facts of the supOU stochastic volatility model introduced in [5]. More background on supOU processes can be found in $[2,4,13,26]$.

In the following, $\mathbb{R}_{-}$denotes the set of negative real numbers and $\mathscr{B}_{b}\left(\mathbb{R}_{-} \times \mathbb{R}\right)$ denotes the bounded Borel sets of $\mathbb{R}_{-} \times \mathbb{R}$.

Definition 2.1 A family $\Lambda=\left\{\Lambda(B): B \in \mathscr{B}_{b}\left(\mathbb{R}_{-} \times \mathbb{R}\right)\right\}$ of real-valued random variables is called a real-valued Lévy basis (infinitely divisible independently scattered random measure) on $\mathbb{R}_{-} \times \mathbb{R}$ if: 
- the distribution of $\Lambda(B)$ is infinitely divisible for all $B \in \mathscr{B}_{b}\left(\mathbb{R}_{-} \times \mathbb{R}\right)$,

- for any $n \in \mathbb{N}$ and pairwise disjoint sets $B_{1}, \ldots, B_{n} \in \mathscr{B}_{b}\left(\mathbb{R}_{-} \times \mathbb{R}\right)$ the random variables $\Lambda\left(B_{1}\right), \ldots, \Lambda\left(B_{n}\right)$ are independent,

- for any sequence of pairwise disjoint sets $B_{n} \in \mathscr{B}_{b}\left(\mathbb{R}_{-} \times \mathbb{R}\right)$ with $n \in \mathbb{N}$ satisfying $\cup_{n \in \mathbb{N}} B_{n} \in \mathscr{B}_{b}\left(\mathbb{R}_{-} \times \mathbb{R}\right)$ the series $\sum_{n=1}^{\infty} \Lambda\left(B_{n}\right)$ converges a.s. and $\Lambda\left(\cup_{n \in \mathbb{N}} B_{n}\right)=$ $\sum_{n=1}^{\infty} \Lambda\left(B_{n}\right)$.

We consider only Lévy bases with characteristic functions of the form

$$
\mathbb{E}(\exp (i u \Lambda(B)))=\exp (\varphi(u) \Pi(B))
$$

for all $u \in \mathbb{R}$ and all $B \in \mathscr{B}_{b}\left(\mathbb{R}_{-} \times \mathbb{R}\right)$, where $\Pi=\pi \times \lambda$ is the product of a probability measure $\pi$ on $\mathbb{R}_{-}$and the Lebesgue measure $\lambda$ on $\mathbb{R}$ and

$$
\varphi(u)=i u \gamma_{0}+\int_{\mathbb{R}_{+}}\left(\mathrm{e}^{i u x}-1\right) v(\mathrm{~d} x)
$$

is the cumulant transform of an infinitely divisible distribution on $\mathbb{R}_{+}$with LévyKhintchine triplet $\left(\gamma_{0}, 0, v\right)$, which is also the characteristic triplet of the underlying Lévy process $L_{t}=\Lambda\left(\mathbb{R}_{-} \times(0, t]\right)$ and $L_{-t}=\Lambda\left(\mathbb{R}_{-} \times(-t, 0)\right)$ for $t \in \mathbb{R}_{+}$(see, e.g., [24] for the relevant background on infinitely divisible distributions and Lévy processes). We call the triplet $\left(\gamma_{0}, \nu, \pi\right)$ the generating triplet. Note that this means that $\gamma_{0} \geq 0, v\left(\mathbb{R} \backslash \mathbb{R}_{+}\right)=0$, and $\int_{|x| \leq 1}|x| v(\mathrm{~d} x)<\infty$.

If $L$ is a pure jump Lévy process with triplet $(0,0, v)$ and jump measure $N(\mathrm{~d} s, \mathrm{~d} x)$, then turning the Poisson point process of jumps in $\mathbb{R} \times \mathbb{R}_{+} \backslash\{0\}$ to one in $\mathbb{R} \times \mathbb{R}_{+} \backslash\{0\} \times$


the jump measure of a Lévy basis with triplet $\left(\gamma_{0}, \nu, \pi\right)$.

In the supOU process defined now, this can be understood as assigning every jump of a Lévy process an individual exponential decay rate. We restrict our attention to positive supOU processes as this is natural when using them to model a variance changing over time.

Theorem 2.2 ([2, 4, 13]) Let $\Lambda$ be an $\mathbb{R}_{+}$-valued Lévy basis on $\mathbb{R}_{-} \times \mathbb{R}$ with generating triplet $\left(\gamma_{0}, v, \pi\right)$. Assume

$$
\int_{|x|>1} \ln (|x|) v(\mathrm{~d} x)<\infty, \text { and }-\int_{\mathbb{R}_{-}} \frac{1}{A} \pi(d A)<\infty .
$$

Then the process $\Sigma=\left(\Sigma_{t}\right)_{t \in \mathbb{R}}$ given by

$$
\Sigma_{t}=\int_{\mathbb{R}_{-}} \int_{-\infty}^{t} \mathrm{e}^{A(t-s)} \Lambda(\mathrm{d} A, \mathrm{~d} s)
$$

is well defined as a Lebesgue integral for all $t \in \mathbb{R}$ and it is stationary. 
Moreover, $\Sigma_{t} \geq 0$ for all $t \in \mathbb{R}$ and the distribution of $\Sigma_{t}$ is infinitely divis-

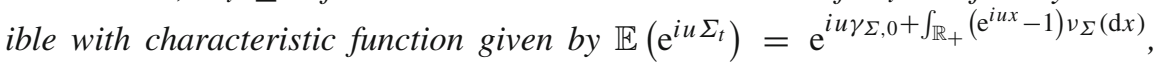
for all $u \in \mathbb{R}$ where

$\gamma_{\Sigma, 0}=\int_{\mathbb{R}_{-}} \int_{0}^{\infty} \mathrm{e}^{A s} \gamma_{0} \mathrm{~d} s \pi(\mathrm{d} A), \nu_{\Sigma}(B)=\int_{\mathbb{R}_{-}} \int_{0}^{\infty} \int_{\mathbb{R}_{+}} \mathbf{1}_{B}\left(\mathrm{e}^{A s} x\right) v(\mathrm{~d} x) \mathrm{d} s \pi(\mathrm{d} A)$,

for all $B \in \mathscr{B}(\mathbb{R})$.

As shown in [4, Theorem 3.12] the supOU process is adapted to the filtration generated by $\Lambda$ and has locally bounded paths. Provided $\pi$ has a finite first moment, one can take a supOU process to have càdlàg paths.

Definition 2.3 Let $W$ be a standard Brownian motion, $a=\left(a_{t}\right)_{t \in \mathbb{R}_{+}}$a predictable real-valued process, $\Lambda$ an $\mathbb{R}_{+}$-valued Lévy basis on $\mathbb{R}_{-} \times \mathbb{R}$ independent of $W$ with generating triplet $\left(\gamma_{0}, v, \pi\right)$ and let $L$ be its underlying Lévy process. Let $\Sigma$ be a non-negative càdlàg supOU process and $\rho \in \mathbb{R}$. Assume that the logarithmic price process $X=\left(X_{t}\right)_{t \in \mathbb{R}_{+}}$is given by

$$
X_{t}=X_{0}+\int_{0}^{t} a_{s} \mathrm{~d} s+\int_{0}^{t} \Sigma_{s}^{\frac{1}{2}} \mathrm{~d} W_{s}+\rho\left(L_{t}-\gamma_{0} t\right)
$$

where $X_{0}$ is independent of $\Lambda$. Then we say that $X$ follows a univariate supOU stochastic volatility model and refer to it by $\operatorname{SV} \sup O U\left(a, \rho, \gamma_{0}, v, \pi\right)$.

In the following, we always use as filtration the one generated by $W$ and $\Lambda$.

In Definition $2.3 X$ is supposed to be the log price of some financial asset and $\rho$ is the typically negative correlation between jumps in the volatility and log asset prices modeling the leverage effect. To ensure that the absolutely continuous drift is completely given by $a_{t}$, we subtract the drift $\gamma_{0}$ from the Lévy process noting that this can be done without loss of generality.

In [5], it has been shown that the model is able to exhibit long-range dependence in the squared log returns. The typical example leading to a polynomial decay of the autocovariance function of the squared returns and to long-range dependence for certain choices of the parameter is to take $\pi$ as a Gamma distribution mirrored at the origin. $[13,26]$ discuss in general which properties of $\pi$ result in long-range dependence.

\section{Martingale Conditions}

Now we assume given a market with a deterministic numeraire (or bond) with price process $\mathrm{e}^{r t}$ for some $r \geq 0$ and a risky asset with price process $S_{t}$.

We want to model the market by a supOU stochastic volatility model under the risk neutral dynamics. Thus, we need to understand when $\hat{S}_{t}=\mathrm{e}^{-r t} \mathrm{e}^{X_{t}}$ is a martingale 
for the filtration $\mathbb{G}=\left(\mathscr{G}_{t}\right)_{t \in \mathbb{R}_{+}}$generated by the Wiener process and the Lévy basis, i.e., $\mathscr{G}_{t}=\sigma\left(\left\{\Lambda(A), W_{s}: s \in[0, t]\right.\right.$ and $\left.\left.A \in \mathscr{B}_{b}\left(\mathbb{R}_{-} \times(-\infty, t]\right)\right\}\right)$ for $t \in \mathbb{R}_{+}$. Implicitly, we understand that the filtration is modified such that the usual hypotheses (see, e.g., [22]) are satisfied.

Theorem 3.1 (Martingale condition) Consider a market as described above. Suppose that

$$
\int_{x>1}\left(\mathrm{e}^{\rho x}-1\right) v(\mathrm{~d} x)<\infty .
$$

If the process $a=\left(a_{t}\right)_{t \in \mathbb{R}_{+}}$satisfies

$$
a_{t}=r-\frac{1}{2} \Sigma_{t}-\int_{\mathbb{R}_{+}}\left(\mathrm{e}^{\rho x}-1\right) v(\mathrm{~d} x),
$$

then the discounted price process $\hat{S}$ is a martingale.

Proof The arguments are straightforward adaptations of the ones in [19, Proposition 2.10] or [20, Sect. 3].

\section{Fourier Pricing in the supOU Stochastic Volatility Model}

Our aim now is to use the Fourier pricing approach in the supOU stochastic volatility model for calculating prices of European derivatives.

\subsection{A Review on Fourier Pricing}

We start with a brief review on the well-known Fourier pricing techniques introduced in $[9,23]$.

Let the price process of a financial asset be modeled as an exponential semimartingale $S=\left(S_{t}\right)_{0 \leq t \leq T}$, i.e., $S_{t}=S_{0} e^{X_{t}}, 0 \leq t \leq T$ where $X=\left(X_{t}\right)_{0 \leq t \leq T}$ is a semimartingale.

Let $r$ be the risk-free interest rate and let us assume that we are directly working under an equivalent martingale measure, i.e., the discounted price process $\hat{S}=\left(\hat{S}_{t}\right)_{0 \leq t \leq T}$ given by $\hat{S}_{t}=S_{0} e^{X_{t}-r t}$ is a martingale.

We call the process $X$ the underlying process and without loss of generality we can assume that $X_{0}=0$. We denote by $s$ minus the logarithm of the initial value of $S$, i.e., $s=-\log \left(S_{0}\right)$.

Let $\hat{f}$ denote the Fourier transform of the function $f$, i.e., $\hat{f}(u)=\int_{\mathbb{R}} e^{i u x} f(x) \mathrm{d} x$. 
Let now $f: \mathbb{R} \rightarrow \mathbb{R}_{+}$be a measurable function that we refer to as the payoff function. Then, the arbitrage-free price of the derivative with payoff $f\left(X_{T}-s\right)$ and maturity $T$ at time zero is the conditional expected discounted payoff under the chosen equivalent martingale measure, i.e., $V_{f}\left(X_{T} ; s\right)=e^{-r t} \mathbb{E}\left(f\left(X_{T}-s\right) \mid \mathscr{G}_{0}\right)$.

The following theorem gives the valuation formula for the price of the derivative paying $f\left(X_{T}-s\right)$ at time $T$.

Theorem 4.1 ([12] Theorem 2.2, Remark 2.3) Let $f: \mathbb{R} \rightarrow \mathbb{R}_{+}$be a payofffunction and let $g_{R}(x)=e^{-R x} f(x)$ for some $R \in \mathbb{R}$ denote the dampened payoff function. Define $\Phi_{X_{T} \mid \mathscr{G}_{0}}(u):=\mathbb{E}\left(e^{u X_{T}} \mid \mathscr{G}_{0}\right), u \in \mathbb{C}$. If

(i) $g_{R} \in L^{1}(\mathbb{R}) \cap L^{\infty}(\mathbb{R}),($ ii $) \Phi_{X_{T} \mid \mathscr{G}_{0}}(R)<\infty$, (iii) $\Phi_{X_{T} \mid \mathscr{G}_{0}}(R+i \cdot) \in L^{1}(\mathbb{R})$,

then $V_{f}\left(X_{T} ; s\right)=\frac{e^{-r t-R s}}{2 \pi} \int_{\mathbb{R}} e^{-i u s} \Phi_{X_{T} \mid \mathscr{G}_{0}}(R+i u) \hat{f}(i R-u) d u$.

It is well known that for a European Call option with maturity $T$ and strike $K>0$ condition $(i)$ is satisfied for $R>1$ and that for the payoff function $f(x)=\max \left(e^{x}-\right.$ $K, 0)=:\left(e^{x}-K\right)^{+}$the Fourier transform is $\hat{f}(u)=\frac{K^{1+i u}}{i u(1+i u)}$ for $u \in \mathbb{C}$ with $\operatorname{Im}(u) \in(1, \infty)$.

In the following, we calculate the characteristic/moment generating function for the supOU SV model and show conditions when the above Fourier pricing techniques are applicable.

\subsection{The Characteristic Function}

Consider the general supOU SV model with drift of the form $a_{t}=\mu+\gamma_{0}+\beta \Sigma_{t}$. Note that then the discounted stock price is a martingale if and only if $\beta=-1 / 2$ and $\mu+\gamma_{0}=r-\int_{\mathbb{R}_{+}}\left(e^{\rho x}-1\right) v(\mathrm{~d} x)$.

Standard calculations as in [19, Theorem 2.5] or [20] give the following result which is the univariate special case of a formula reported in [4, Sect. 5.2].

Theorem 4.2 Let $X_{0} \in \mathbb{R}$ and let the log-price process $X$ follow a supOU SV model of the above form. Then, for every $t \in \mathbb{R}_{+}$and for all $u \in \mathbb{R}$ the characteristic function of $X_{t}$ given $\mathscr{G}_{0}$ is given by

$$
\begin{aligned}
\Phi_{X_{t} \mid \mathscr{G}_{0}}(i u)= & \mathbb{E}\left(e^{i u X_{t}} \mid \mathscr{G}_{0}\right) \\
= & \exp \left\{i\left(u\left(X_{0}+\mu t\right)+\left(u \beta+\frac{i}{2} u^{2}\right) \int_{\mathbb{R}_{-}} \int_{-\infty}^{0} \frac{1}{A}\left(e^{A(t-s)}-e^{-A s}\right) \Lambda(\mathrm{d} A, \mathrm{~d} s)\right)\right. \\
& \left.+\int_{\mathbb{R}_{-}} \int_{0}^{t} \varphi\left(\frac{e^{A(t-s)}}{A}\left(u \beta+\frac{i}{2} u^{2}\right)-\left(\frac{1}{A}\left(u \beta+\frac{i}{2} u^{2}\right)-\rho u\right)\right) \mathrm{d} s \pi(\mathrm{d} A)\right\} .
\end{aligned}
$$


Note that in contrast to the case of the OU-type stochastic volatility model, where $(X, \Sigma)$ is a strong Markov process, in the supOU stochastic volatility model $\Sigma$ is not Markovian. Thus, conditioning on $X_{0}$ and $\Sigma_{0}$ is not equivalent to conditioning upon $\mathscr{G}_{0}$. Therefore, $\Phi_{X_{t} \mid \mathscr{G}_{0}}(i u)$ is not simply a function of $X_{0}, \Sigma_{0}$. Instead, the whole past of the Lévy basis enters via the $\mathscr{G}_{0}$-measurable

$$
z_{t}:=\int_{\mathbb{R}_{-}} \int_{-\infty}^{0} \frac{1}{A}\left(e^{A(t-s)}-e^{-A s}\right) \Lambda(\mathrm{d} A, \mathrm{~d} s),
$$

which has a similar role as the initial volatility $\Sigma_{0}$ in the OU-type stochastic volatility model. Like $\Sigma_{0}$ in the OU-type models, $z_{t}$ can be treated as an additional parameter to be determined when calibrating the model to market option prices. We can immediately see that thus the number of parameters to be estimated increases with each additional maturity. As it will become clear later, the following observation is important.

Lemma 4.3 $z_{t_{1}} \leq z_{t_{2}}$, for all $t_{1}, t_{2} \in \mathbb{R}_{+}$such that $t_{1} \leq t_{2}$.

Proof For $t \in \mathbb{R}_{+}$and $s \leq t$ we have $\frac{1}{A}\left(e^{A(t-s)}-e^{-A s}\right)=\frac{e^{-A s}}{A}\left(e^{A t}-1\right)$ and for $t_{1} \leq t_{2}$ one sees $e^{A t_{2}}-1 \leq e^{A t_{1}}-1 \leq 0$ since $A<0$. This implies that for $s \leq t_{1} \leq t_{2} \frac{e^{-A s}}{A}\left(e^{A t_{1}}-1\right) \leq \frac{e^{-A s}}{A}\left(e^{A t_{2}}-1\right)$ and thus $z_{t_{1}} \leq z_{t_{2}}$.

\subsection{Regularity of the Moment Generating Function}

In order to apply Fourier pricing, we now show where the moment generating function $\Phi_{X_{T} \mid \mathscr{G}_{0}}$ is analytic.

Let $\theta_{L}(u)=\gamma_{0} u+\int_{\mathbb{R}_{+}}\left(e^{u x}-1\right) v(\mathrm{~d} x)$ be the cumulant transform of the Lévy basis (or rather its underlying subordinator). If $\int_{x \geq 1} e^{r x} v(\mathrm{~d} x)<\infty$ for all $r \in$ $\mathbb{R}$ such that $r<\varepsilon$ for some $\varepsilon>0$, then the function $\theta_{L}$ is analytic in the open set $S_{L}:=\{z \in \mathbb{C}: \operatorname{Re}(z)<\varepsilon\}$, as can be seen, e.g., from the arguments at the start of the proof of [19, Lemma 2.7].

Theorem 4.4 Let the measure v satisfy

$$
\int_{x \geq 1} e^{r x} v(\mathrm{~d} x)<\infty \text { for all } r \in \mathbb{R} \text { such that } r<\varepsilon
$$

for some $\varepsilon>0$. Then the function $\Theta(u)=\int_{\mathbb{R}_{-}} \int_{0}^{t} \theta_{L}\left(u f_{u}(A, s)\right) \mathrm{d} s \pi(\mathrm{d} A)$ is analytic on the open strip 


$$
S:=\{u \in \mathbb{C}, \quad|\operatorname{Re}(u)|<\delta\} \quad \text { with } \delta:=-|\beta|-\frac{|\rho|}{t}+\sqrt{\Delta}
$$

where $\Delta:=\left(|\beta|+\frac{|\rho|}{t}\right)^{2}+\frac{2 \varepsilon}{t}$.

The rough idea of the proof is similar to [19, Theorem 2.8], but the fact that we now integrate over the mean reversion parameter adds significant difficulty, as now bounds independent of the mean reversion parameter need to be obtained and a very general holomorphicity result for integrals has to be employed.

Proof Define

$$
f_{u}(A, s)=\mathbf{1}_{[0, t]}(s)\left(\frac{e^{A(t-s)}}{A}\left(\beta+\frac{u}{2}\right)-\left(\frac{1}{A}\left(\beta+\frac{u}{2}\right)-\rho\right)\right) .
$$

We first determine $\delta>0$ such that for all $u \in \mathbb{R}$ with $|u|<\delta$ it holds that $\left|u f_{u}(A, s)\right|<\varepsilon$. We have

$$
\left|u f_{u}(A, s)\right| \leq\left|\frac{e^{A(t-s)}-1}{A}\right|\left(|\beta||u|+\frac{u^{2}}{2}\right)+|\rho||u|
$$

by the triangle inequality. In order to find the upper bound for the latter term, we first note that elementary analysis shows

$$
\left|\frac{e^{A(t-s)}-1}{A}\right| \leq t
$$

for all $A<0$ and $s \in[0, t]$. Thus, we have to find $\delta>0$ such that $\left|u f_{u}(A, s)\right| \leq$ $t\left(|\beta||u|+\frac{u^{2}}{2}\right)+|\rho||u|<\varepsilon$, for all $u \in \mathbb{R}$ with $|u|<\delta$, i.e., to find the solutions of the quadratic equation

$$
\frac{t}{2} u^{2}+(t|\beta|+|\rho|)|u|-\varepsilon=0 .
$$

Since for $u=0$ the sign of (9) is negative, i.e., (9) is equal to $-\varepsilon$, we know that there exist one positive and one negative solution. The positive one is $\delta$ as given in (5).

Now let $u \in S$, i.e., $u=v+i w$ with $v, w \in \mathbb{R},|v|<\delta$. Observe that $\operatorname{Re}\left(u f_{u}(A, s)\right)=v f_{v}(A, s)-\frac{w^{2}}{2}\left(\frac{e^{A(t-s)}-1}{A}\right)$ and $\frac{e^{A(t-s)}-1}{A} \geq 0$ for all $s \in[0, t]$ and $A<0$. Hence, $\operatorname{Re}\left(u f_{u}(A, s)\right) \leq v f_{v}(A, s)$. This implies that

$$
\int_{x \geq 1} e^{\operatorname{Re}\left(u f_{u}(A, s)\right) x} v(\mathrm{~d} x) \leq \int_{x \geq 1} e^{v f_{v}(A, s) x} v(\mathrm{~d} x)<\infty
$$


due to $\left|v f_{v}(A, s)\right|<\varepsilon$ for $|v|<\delta$ and condition (4). Hence for $u \in S$ the function $\theta_{L}\left(u f_{u}(A, s)\right)=\gamma_{0} u f_{u}(A, s)+\int_{\mathbb{R}_{+}}\left(e^{u f_{u}(A, s) x}-1\right) v(\mathrm{~d} x)$ is well defined. $u f_{u}(A, s)$ is a polynomial of $u$ and thus it is an analytic function in $\mathbb{C}$, for all $s \in[0, t]$ and $A<0$. The function $\theta_{L}$ is analytic in the set $S_{L}=\{z \in \mathbb{C}: \quad|\operatorname{Re}(z)|<\varepsilon\}$.

Thus, the function $\theta_{L}\left(u f_{u}(A, s)\right)$ is analytic in $S$, for all $s \in[0, t]$ and $A<0$. By the holomorphicity theorem for parameter dependent integrals (see, e.g., [15]), we can conclude that $\int_{0}^{t} \theta_{L}\left(u f_{u}(A, s)\right) \mathrm{d} s$ is analytic in $S$, for all $A<0$.

Defining $\varphi(u, A):=\int_{0}^{t} \theta_{L}\left(u f_{u}(A, s)\right) \mathrm{d} s$ we now apply [17] to prove that $\Theta(u)=$ $\int_{\mathbb{R}_{-}} \int_{0}^{t} \theta_{L}\left(u f_{u}(A, s)\right) \mathrm{d} s \pi(\mathrm{d} A)=\int_{\mathbb{R}_{-}} \varphi(u, A) \pi(\mathrm{d} A)$ is analytic in $S$. Its conditions $A_{1}$ and $A_{2}$ are obviously satisfied. It remains to prove that condition $A_{3}$ holds, i.e., that $\int_{\mathbb{R}_{-}}|\varphi(u, A)| \pi(\mathrm{d} A)$ is locally bounded. First, observe that

$$
\begin{aligned}
\left|\theta_{L}\left(u f_{u}(A, s)\right)\right| \leq & \left|\gamma_{0} u f_{u}(A, s)\right|+\int_{x \leq 1}\left|e^{u f_{u}(A, s) x}-1\right| v(\mathrm{~d} x) \\
& +\int_{x>1}\left|e^{u f_{u}(A, s) x}-1\right| v(\mathrm{~d} x)
\end{aligned}
$$

Using (8), we can bound the first summand in (10) by:

$$
\left|\gamma_{0} u f_{u}(A, s)\right| \leq\left|\gamma_{0}\right|\left(t\left(|\beta||u|+\frac{|u|^{2}}{2}\right)+|\rho||u|\right)=: B_{1}(u) .
$$

For the second summand, using Taylor's theorem we have that $\left|e^{u f_{u}(A, s) x}-1\right| \leq$ $\left|u f_{u}(A, s)\right||x|+O\left(\left|u f_{u}(A, s)\right|^{2}|x|^{2}\right)$. Since $\left|u f_{u}(A, s)\right| \leq t\left(|\beta||u|+\frac{|u|^{2}}{2}\right)+|\rho||u|$, for the remainder term of Taylor's formula we have

$$
O\left(\left|u f_{u}(A, s)\right|^{2}|x|^{2}\right) \leq O\left(\left|t\left(|\beta||u|+\frac{|u|^{2}}{2}\right)+\right| \rho|| u||^{2}|x|^{2}\right)
$$

where the latter term converges to zero as $x \rightarrow 0$. If we define

$$
K(u):=t\left(|\beta||u|+\frac{|u|^{2}}{2}\right)+|\rho||u|
$$

we obtain that

$$
\int_{x \leq 1}\left|e^{u f_{u}(A, s) x}-1\right| v(\mathrm{~d} x) \leq K(u) \int_{x \leq 1} x v(\mathrm{~d} x)+\int_{x \leq 1} O\left(K(u)^{2}|x|^{2}\right) v(\mathrm{~d} x)=: B_{2}(u),
$$

which is finite due to the properties of the measure $v$. 
Let $S_{n}:=\{\mathbb{C} \ni u=v+i w: \quad|v| \leq \delta-1 / n\} \subseteq S$. Since the function $v f_{v}(A, s)$ is continuous on the compact set $V_{n}=\{v \in \mathbb{R}:|v| \leq \delta-1 / n\}$, it attains its minimum and maximum on that set, i.e., there exists $v^{*} \in V_{n}$ such that $v f_{v}(A, s) \leq$ $v^{*} f_{v^{*}}(A, s) \leq\left|v^{*} f_{v^{*}}(A, s)\right|=: K_{n}(u)$ for all $v \in V_{n}$. Note that $v^{*} \in V_{n}$ implies that $K_{n}(u)<\varepsilon$. Since $\operatorname{Re}\left(u f_{u}(A, s)\right) \leq v f_{v}(A, s)$ and $\left|e^{u f_{u}(A, s) x}\right|=e^{\operatorname{Re}\left(u f_{u}(A, s)\right) x} \leq$ $e^{K_{n}(u) x}$, it follows that

$$
\int_{x>1}\left|e^{u f_{u}(A, s) x}-1\right| v(\mathrm{~d} x) \leq \int_{x>1} e^{K_{n}(u) x} v(\mathrm{~d} x)+\int_{x>1} v(\mathrm{~d} x)=: B_{3, n}(u),
$$

which is finite due to (4) and the properties of the measure $v$.

Since $B_{1}(u), B_{2}(u)$, and $B_{3, n}(u)$ do not depend neither on $s$ nor on $A$, we have $|\varphi(u, A)| \leq t\left(B_{1}(u)+B_{2}(u)+B_{3, n}(u)\right)$ and

$$
\int_{\mathbb{R}_{-}} t\left(B_{1}(u)+B_{2}(u)+B_{3, n}(u)\right) \pi(\mathrm{d} A)=t\left(B_{1}(u)+B_{2}(u)+B_{3, n}(u)\right)<\infty,
$$

so the function $t\left(B_{1}(u)+B_{2}(u)+B_{3, n}(u)\right)$ is integrable with respect to $\pi$. Since $\varphi(u, A)$ is analytic and thus a continuous function on $S_{n}$, for all $A<0$, it also holds that $|\varphi(u, A)|$ is continuous on $S_{n}$, for all $A<0$. By the dominated convergence theorem, it follows that $\int_{\mathbb{R}_{-}}|\varphi(u, A)| \pi(\mathrm{d} A)$ is continuous and thus a locally bounded function on $S_{n}$. Since $n \in \mathbb{N}$ was arbitrary, it follows that the function is continuous and locally bounded on $S$, which completes the proof.

Now, we can easily give conditions ensuring that (ii) in Theorem 4.1 is satisfied.

Corollary 4.5 Let $\int_{x \geq 1} e^{r x} v(\mathrm{~d} x)<\infty$ for all $r \in \mathbb{R}$ such that $r<\varepsilon$ for some $\varepsilon>0$. Then the moment generating function $\Phi_{X_{T} \mid G_{0}}$ is analytic on the open strip $S:=\{u \in \mathbb{C}:|\operatorname{Re}(u)|<\delta\}$ with $\delta:=-|\beta|-\frac{|\rho|}{T}+\sqrt{\Delta}$ where $\Delta:=\left(|\beta|+\frac{|\rho|}{T}\right)^{2}+$ $\frac{2 \varepsilon}{T}$. Furthermore,

$$
\begin{aligned}
& \Phi_{X_{T} \mid \mathscr{G}_{0}}(u)= \\
& \quad \exp \left\{u\left(X_{0}+\mu T\right)+\left(u \beta+\frac{1}{2} u^{2}\right) \int_{\mathbb{R}_{-}} \int_{-\infty}^{0} \frac{1}{A}\left(e^{A(T-s)}-e^{-A s}\right) \Lambda(\mathrm{d} A, \mathrm{~d} s)+\Theta(u)\right\}
\end{aligned}
$$

for all $u \in S$.

Proof Follows from Theorems 4.2 and 4.4 noting that an analytic function is uniquely identified by its values on a line and [19, Lemma A.1].

Very similar to [19, Theorem 6.11], we can now prove that also condition (iii) in Theorem 4.1 is satisfied for the supOU SV model.

Theorem 4.6 If $u \in \mathbb{C}, u=v+i w$ and $u \in S$ as defined in Theorem 4.4, then the map 


$$
w \mapsto \Phi_{X_{T} \mid \mathscr{G}_{0}}(v+i w)
$$

is absolutely integrable.

\section{Examples}

\subsection{Concrete Specifications}

If we want to price a derivative by Fourier inversion, then this means in the supOU SV model that we have to calculate the inverse Fourier transform by numerical integration and inside this the double integral in $\Theta(u)=\int_{\mathbb{R}_{-}} \int_{0}^{t} \theta_{L}\left(u f_{u}(A, s)\right) \mathrm{d} s \pi(\mathrm{d} A)$. If we want to calibrate our model to market data, the optimizer will repeat this procedure very often and so it is important to consider specifications where at least some of the integrals can be calculated analytically.

Actually, it is not hard to see that one can use the standard specifications for $v$ of the OU-type stochastic volatility model (see [3, 11, 20, 25]) which are named after the resulting stationary distribution of the OU-type processes.

As in the case of a $\Gamma$-OU process we can choose the underlying Lévy process to be a compound Poisson process with the characteristic triplet $\left(\gamma_{0}, 0, a b e^{-b x} \mathbf{1}_{\{x>0\}} \mathrm{d} x\right)$ with $a, b>0$ where abusing notation we specified the Lévy measure by its density. Furthermore, we assume that $A$ follows a "negative" $\Gamma$-distribution, i.e., that $\pi$ is the distribution of $B R$, where $B \in \mathbb{R}_{-}$and $R \sim \Gamma(\alpha, 1)$ with $\alpha>1$ which is the specification typically used to obtain long memory/a polynomial decay of the autocorrelation function. We refer to this specification as the $\Gamma$-supOU SV model.

Using (6) we have

$$
\Theta(u)=u \int_{\mathbb{R}_{-}} \int_{0}^{t} \gamma_{0} f_{u}(A, s) \mathrm{d} s \pi(\mathrm{d} A)+\int_{\mathbb{R}_{-}} \int_{0}^{t} \int_{\mathbb{R}_{+}}\left(e^{u f_{u}(A, s) x}-1\right) \nu(\mathrm{d} x) \mathrm{d} s \pi(\mathrm{d} A) .
$$

For the first summand in $\Theta(u)$ we see

$$
\begin{aligned}
& u \int_{\mathbb{R}_{-}} \int_{0}^{t} \gamma_{0} f_{u}(A, s) \mathrm{d} s \pi(\mathrm{d} A)=\gamma_{0}(\underbrace{\iint_{\mathbb{R}_{-}}^{t} \frac{e^{A(t-s)}}{A}\left(u \beta+\frac{u^{2}}{2}\right) \mathrm{d} s \pi(\mathrm{d} A)}_{I_{1}} \\
& -\underbrace{\int_{\mathbb{R}_{-}} \int_{0}^{t} \frac{1}{A}\left(u \beta+\frac{u^{2}}{2}\right) \mathrm{d} s \pi(\mathrm{d} A)}_{I_{2}}+\underbrace{\int_{\mathbb{R}_{-}} \int_{0}^{t} \rho u \mathrm{~d} s \pi(\mathrm{d} A)}_{I_{3}}) .
\end{aligned}
$$


For the three parts, we can now show:

$$
\begin{aligned}
& I_{1}=\left(u \beta+\frac{u^{2}}{2}\right) \frac{(1-B t)^{2-\alpha}-1}{B^{2}(\alpha-1)(\alpha-2)} \text { if } \alpha \neq 2, \\
& I_{1}=-\frac{\left(u \beta+\frac{u^{2}}{2}\right)}{B^{2}} \ln (1-B t) \text { if } \alpha=2, \\
& I_{2}=\frac{t\left(u \beta+\frac{u^{2}}{2}\right)}{B(\alpha-1)}, \quad I_{3}=\rho u \int_{0}^{t} \int_{\mathbb{R}_{-}} \mathrm{d} s \pi(\mathrm{d} A)=\rho u t .
\end{aligned}
$$

Furthermore setting $C(A):=\frac{1}{A}\left(u \beta+\frac{u^{2}}{2}\right)-\rho u$ one obtains for the second summand in $\Theta$

$$
\begin{aligned}
& \int_{\mathbb{R}_{-}} \int_{0}^{t} \int_{\mathbb{R}_{+}}\left(e^{u f_{u}(A, s) x}-1\right) a b e^{-b x} \mathrm{~d} x \mathrm{~d} s \pi(\mathrm{d} A) \\
& \quad=a \int_{\mathbb{R}_{-}} \frac{1}{A(b+C(A))}\left(b \ln \left(\frac{b-\rho u}{b-\frac{e^{A t}}{A}\left(u \beta+\frac{u^{2}}{2}\right)+C(A)}\right)-A C(A) t\right) \pi(\mathrm{d} A) .
\end{aligned}
$$

Unfortunately, we have been unable to obtain a more explicit formula for this integral, and so it has to be calculated numerically. In our example later on we have used the standard Matlab command "integral" for this. Note that the well-behavedness of this numerical integration depends on the choice of $\pi$. For our choice, $\pi$ being a negative Gamma distribution implies roughly (i.e., up to a power) an exponentially fast decaying integrand for $A \rightarrow \infty$, whereas the behavior at zero appears to be hard to determine.

We can also choose the underlying Lévy process as in an IG-OU model with parameters $\delta$ and $\gamma$, while keeping the choice of the measure $\pi$ the same. In this case, we have $v(\mathrm{~d} x)=\frac{1}{2 \sqrt{2 \pi}} \delta\left(x^{-1}+\gamma^{2}\right) x^{-\frac{1}{2}} \exp \left(-\frac{1}{2} \gamma^{2} x\right) \mathbf{1}_{\{x>0\}} \mathrm{d} x$ and the only difference compared to the previous case is in the calculation of the triple integral which also can be partially calculated analytically so that only a one-dimensional numerical integration is necessary.

\subsection{Calibration and an Illustrative Example}

In this chapter, we calibrate the $\Gamma$-supOU SV model to market prices of European plain vanilla call options written on the DAX.

Let $t_{1}, t_{2}, \ldots, t_{M}$ be the set of different times to maturity (in increasing order) for which we have market option prices. The parameters to be determined by calibration 
Table 1 Calibrated supOU SV model parameters for DAX data of August 19, 2013

\begin{tabular}{l|l|l|l|l|l|l|l}
\hline & $\rho$ & $a$ & $b$ & $B$ & $\alpha$ & $\gamma_{0}$ & \\
\cline { 2 - 8 } & -10.8797 & 0.2225 & 29.4025 & -0.0004 & 4.3632 & 0.0000 & \\
\hline$z_{t_{1}}$ & $z_{t_{2}}$ & $z_{t_{3}}$ & $z_{t_{4}}$ & $z_{t_{5}}$ & $z_{t_{6}}$ & $z_{t_{7}}$ & $z_{t_{8}}$ \\
\hline 0.0012 & 0.0026 & 0.0038 & 0.0054 & 0.0093 & 0.0136 & 0.0225 & 0.0328 \\
\hline
\end{tabular}

are $\left(\rho, a, b, B, \alpha, \gamma_{0}, z_{t_{1}}, \ldots, z_{t_{M}}\right)$, where $\rho$ describes the leverage, $a$ and $b$ are parameters of the measure $\nu, B$, and $\alpha$ are parameters of the measure $\pi$ and $\gamma_{0}$ is the drift parameter. Finally, $z_{t_{1}}, \ldots, z_{t_{M}}$ are $z_{t_{i}}=\int_{\mathbb{R}_{-}} \int_{-\infty}^{0} \frac{1}{A}\left(e^{A\left(t_{i}-s\right)}-e^{-A s}\right) \Lambda(\mathrm{d} A, \mathrm{~d} s)$, $i=1, \ldots, M$.

We calibrate by minimizing the root mean squared error between the BlackScholes implied volatilities corresponding to market and model prices, i.e., RMSE $=$ $\sqrt{\sum_{i=1}^{M} \sum_{j=1}^{N_{i}}\left(\operatorname{blsimpv}\left(C_{i j}^{M}\right)-\operatorname{blsimpv}\left(C_{i j}\right)\right)^{2}} / \sum_{i=1}^{M} N_{i}$, where $M$ is the number of different times to maturity, $N_{i}$ is the number of options for each maturity, $\left\{C_{i j}^{M}\right\}$ is the set of market prices and $\left\{C_{i j}\right\}$ is the set of model prices, $i=1, \ldots, N_{M}$, $j=1, \ldots, M$. Of course, minimizing the difference between Black-Scholes implied volatilities is just one possible choice for the objective function. We note that this data example is only supposed to be an illustrative proof of concept and that using other objective functions including in particular weights for the different options should improve the results.

We use closing prices of 200 DAX options on August 19, 2013. The level of DAX on that day was 8366.29. The data source was Bloomberg Finance L.P. and all the options were listed on EUREX.

For the instantaneous risk-free interest rate, we used the 3-month LIBOR rate, which was $0.15173 \%$. The maturities of the options were $31,59,87,122,213,304$, 486 , and 668 days. The calibration procedure was performed in MATLAB. To avoid being stuck in local minima the calibration was run several times with different initial values and the overall minimum RMSE was taken.

The implied parameters from the calibration procedure are given in Table 1. The fit is good: The RMSE is 0.0046. We plot market against model Black-Scholes implied volatilities in Fig. 1. Although the RMSE is very low and in plots of market against fitted model prices (not shown here) one sees basically no differences, Fig. 1 shows that our model fits the implied volatilities for medium and long maturities very well, but the quality of the fit for shorter maturities is lower.

The vector of the parameters $\left\{z_{i}\right\}_{i=1, \ldots, M}$ is indeed increasing with maturity (cf. Lemma 4.3), although we actually refrained from including this restriction into our optimization problem. The autocorrelation function of the $\Gamma$-supOU model exhibits long memory for $\alpha \in(1,2)$ (cf. [26, Sect. 2.2]). Since the calibration returns $\alpha=4.3632$, our market data are in line with a rather slow polynomial decay of the autocorrelation function, which is in contrast to the exponential decay of the autocorrelation function in the OU-type SV model, but the calibrated model does not 

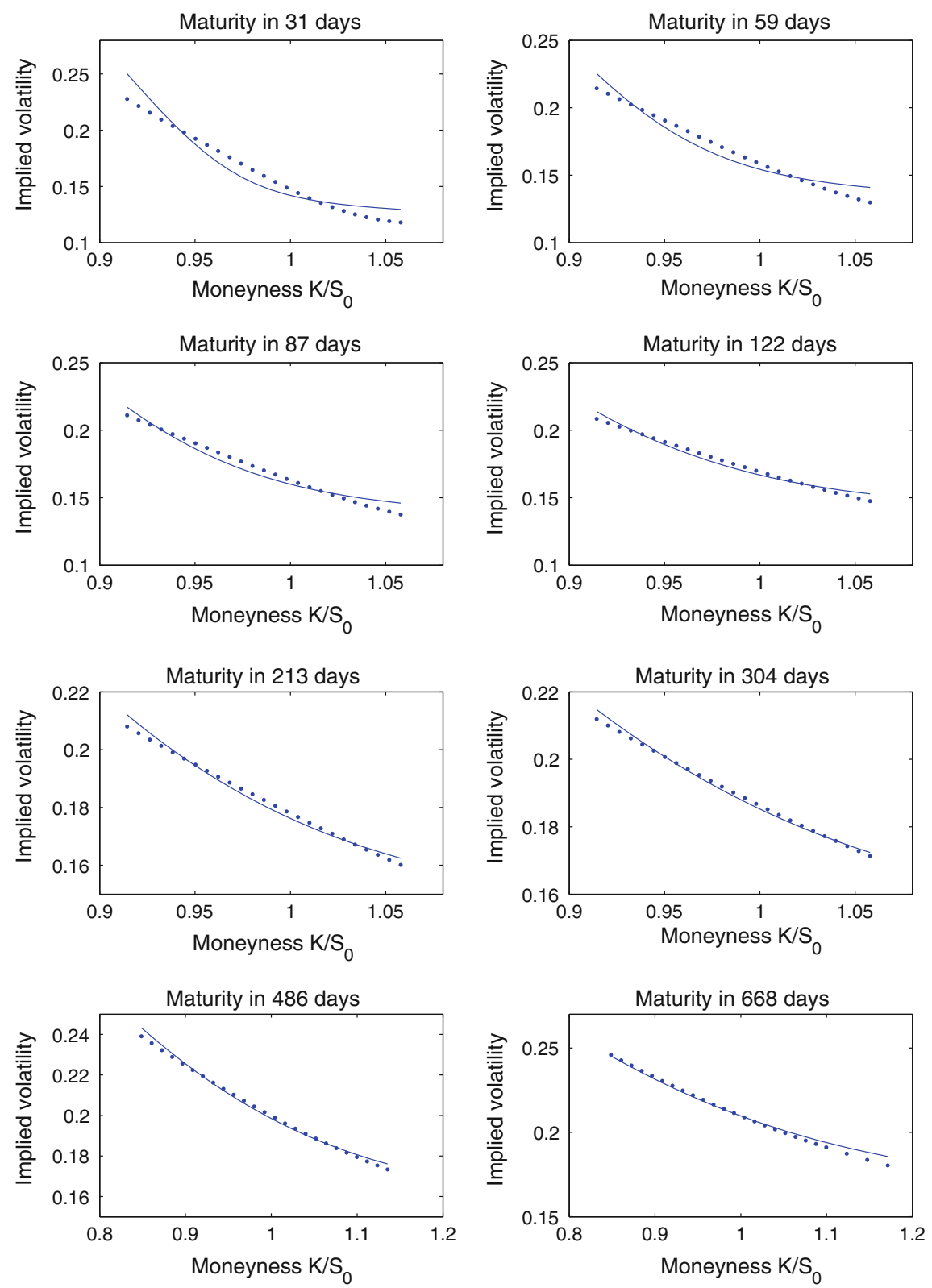

Fig. 1 Calibration of the supOU model to call options on DAX: The Black-Scholes implied volatilities. The implied volatilities from market prices are depicted by a $d o t$, the implied volatilities from model prices by a solid line 
exhibit long memory. One should be very careful not to overinterpret these findings, as no confidence intervals/hypothesis tests are available in connection with such a standard calibration.

The leverage parameter $\rho$ is negative, which implies a negative correlation between jumps in the volatility and returns. Hence, the typical leverage effect is present. The drift parameter of the underlying Lévy basis $\gamma_{0}$ is estimated to be practically zero. So our calibration suggests that a driftless pure jump Lévy basis may be quite adequate to use.

Let us briefly turn to a comparison with the OU-type stochastic volatility model (cf. [19] or [20]) noting that a detailed comparison with various other models is certainly called for, but beyond the scope of the present paper. For some $\beta<0$ looking at a sequence of $\Gamma$-supOU models with $\alpha_{n}=n, B_{n}=\beta / n$ and all others parameters fixed, shows that the mean reversion probability measures $\pi_{n}$ converge weakly to the delta distribution at $\beta$. So the OU model is in some sense a limiting case of the supOU model. However, the limiting model is very different from all approximating models, as it is Markovian, has the same decay rate for all jumps, whereas the approximating supOU models have all negative real numbers as possible decay rates for individual jumps. This implies that in connection with real data the behavior of the OU and the supOU model can well be rather different. Calibrating a $\Gamma$-OU model to our DAX data set (so the only parameter now different is $\pi$, which is a Dirac measure) returns actually a globally better fit (the RMSE is 0.0037). Looking at the plots of market against model implied volatilities they all look quite similar (Fig. 2 shows only the last four largest maturities) to the ones in Fig. 1, although the fit for the early maturities is definitely better when looking closely. Yet, there is one big exception, the last maturity, where the supOU model fits much better. Whereas the rate of the underlying compound Poisson process is $a=0.2225$ in the supOU model, it is 1.2671 in the OU model. The mean of the decay rates is -0.0017 in the supOU model and the decay rate of the OU case is -1.3906 . Noting that the standard deviation of the decay rates is 0.0008 in the supOU model, the two calibrated models are indeed in many respects rather different.

Remark 5.1 (How to price options with general maturities?) After having calibrated a model to observed liquid market prices one often wants to use it to price other (exotic) derivatives. Looking at a European derivative with payoff $f\left(S_{T}\right)$ for some measurable function $f$ and maturity $T>0$, one soon realizes that we can only obtain its price directly if $T \in\left\{t_{1}, t_{2}, \ldots, t_{M}\right\}$, as only then we know $z_{T}$, thus the characteristic function $\Phi_{X_{T} \mid \mathscr{G}_{0}}$ and therefore the distribution of the price process at time $T$ conditional on our current information $\mathscr{G}_{0}$. This is not desirable and the problem is that we assume that we know $\mathscr{G}_{0}$ in theory, but we have only limited information in the market prices which we can use to get only parts of the information in $\mathscr{G}_{0}$.

It seems that to get $z_{t}$ for all $t \in \mathbb{R}_{+}$one needs to really know the whole past of $\Lambda$, i.e., all jumps before time 0 and the associated times and decay rates. This is clearly not feasible. A detailed analysis on the dependence of $z_{t}$ on $t$ is beyond the scope of this paper. But we briefly want to comment on possible ad hoc solutions 

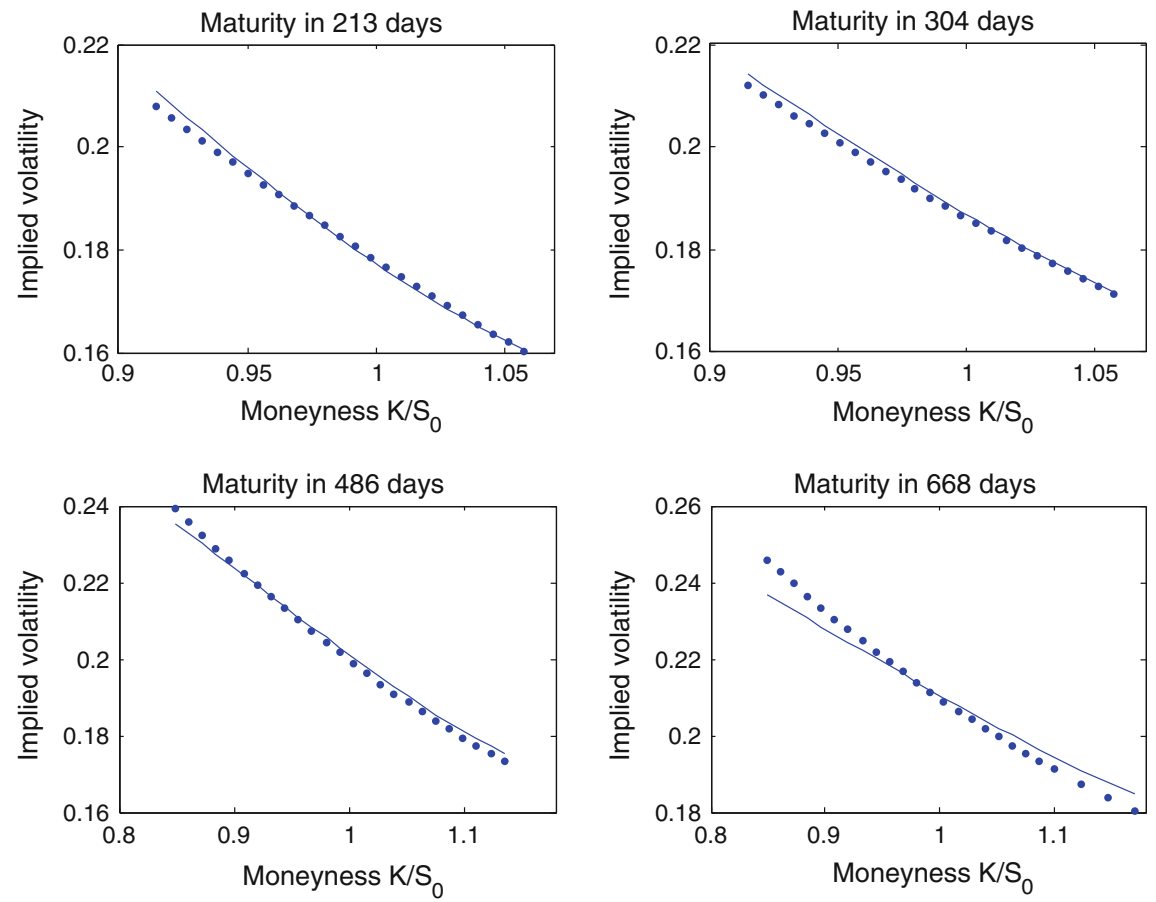

Fig. 2 Calibration of the OU model to call options on DAX: The implied volatilities from market prices are depicted by a dot, the implied volatilities from model prices by a solid line. Last four maturities only

to "estimate" $z_{T}$ based on $\left\{z_{t_{i}}\right\}_{i=1, \ldots, M}$. The first one is to either interpolate or fit a parametric curve $t \mapsto z_{t}$ to the "observed" $\left\{z_{t_{i}}\right\}_{i=1, \ldots, M}$. If one also ensures the decreasingness in $t$ in this procedure, one should get a reasonable approximation, especially when the grid $\left\{t_{i}\right\}_{i=1, \ldots, M}$ is fine and one considers maturities in $\left[t_{1}, t_{M}\right]$.

From the probabilistic point of view, one wants to compute $E\left(z_{T} \mid\left\{z_{t_{i}}\right\}_{i=1, \ldots, M}\right)$ for $T \notin\left\{t_{1}, t_{2}, \ldots, t_{M}\right\}$. Whether and how this conditional expectation can be calculated, is again a question for future investigations. But what one can calculate easily is the best (in the $L^{2}$ sense) linear predictor of $z_{T}$ given $\left\{z_{t_{i}}\right\}_{i=1, \ldots, M}$. One simply needs to straightforwardly adapt standard time series techniques (like the innovations algorithm or linear $L^{2}$ filtering, see, e.g., [8]) noting that one has

$\operatorname{cov}\left(z_{t}, z_{u}\right)=\int_{\mathbb{R}_{-}} \int_{\mathbb{R}_{-}} \frac{e^{-2 A s}}{A^{2}}\left(e^{A t}-1\right)\left(e^{A u}-1\right) \mathrm{d} s \pi(\mathrm{d} A) \int_{\mathbb{R}_{+}} x^{2} v(\mathrm{~d} x) \forall t, u \in \mathbb{R}_{+}$.

Open Access This chapter is distributed under the terms of the Creative Commons Attribution Noncommercial License, which permits any noncommercial use, distribution, and reproduction in any medium, provided the original author(s) and source are credited. 


\section{References}

1. Bannör, K.F., Scherer, M.: A BNS-type stochastic volatility model with two-sided jumps with applications to FX options pricing. Wilmott 2013, 58-69 (2013)

2. Barndorff-Nielsen, O.: Superposition of Ornstein-Uhlenbeck type processes. Theory Probab. Appl. 45, 175-194 (2001)

3. Barndorff-Nielsen, O., Shephard, N.: Non-Gaussian Ornstein-Uhlenbeck-based models and some of their uses in financial economics (with discussion). J. R. Stat. Soc. B Stat. Methodol. 63, 167-241 (2001)

4. Barndorff-Nielsen, O., Stelzer, R.: Multivariate supOU processes. Ann. Appl. Probab. 21(1), 140-182 (2011)

5. Barndorff-Nielsen, O., Stelzer, R.: The multivariate supOU stochastic volatility model. Math. Finance 23, 275-296 (2013)

6. Bender, C., Sottinen, T., Valkeila, E.: Arbitrage with fractional Brownian motion? Theory Stoch. Process. 13(1-2), 23-34 (2007)

7. Björk, T., Hult, H.: A note on Wick products and the fractional Black-Scholes model. Finance Stoch. 9(2), 197-209 (2005)

8. Brockwell, P.J., Davis, R.A.: Time Series: Theory and Methods, vol. 2. Springer, New York (1991)

9. Carr, P., Madan, D.B.: Option valuation using the fast Fourier transform. J. Comput. Finance 2, 61-73 (1999)

10. Cont, R.: Empirical properties of asset returns: stylized facts and statistical issues. Quant. Finance 1, 223-236 (2001)

11. Cont, R., Tankov, P.: Financial Modelling with Jump Processes. CRC Financial Mathematical Series. Chapman \& Hall, London (2004)

12. Eberlein, E., Glau, K., Papapantoleon, A.: Analysis of Fourier transform valuation formulas and applications. Appl. Math. Finance 17, 211-240 (2010)

13. V, Fasen, Klüppelberg, C.: Extremes of supOU processes. In: Benth, F.E., Di Nunno, G., Lindstrom, T., Øksendal, B., Zhang, T. (eds.) Stochastic Analysis and Applications: The Abel Symposium 2005. Abel Symposia, vol. 2, pp. 340-359. Springer, Berlin (2007)

14. Guillaume, D.M., Dacorogna, M.M., Davé, R.D., Müller, U.A., Olsen, R.B., Pictet, O.V.: From the bird's eye to the microscope: a survey of new stylized facts of the intra-daily foreign exchange markets. Finance Stoch. 1, 95-129 (1997)

15. Königsberger, K.: Analysis 2. Springer, Heidelberg (2004)

16. Marquardt, T.: Fractional Lévy processes with an application to long memory moving average processes. Bernoulli 12, 1099-1126 (2006)

17. Mattner, L.: Complex differentiation under the integral. Nieuw Archief voor Wiskunde 5/2(2), 32-35 (2001)

18. Moser, M., Stelzer, R.: Tail behavior of multivariate Lévy driven mixed moving average processes and related stochastic volatility models. Adv. Appl. Probab. 43, 1109-1135 (2011)

19. Muhle-Karbe, J., Pfaffel, O., Stelzer, R.: Option pricing in multivariate stochastic volatility models of OU type. SIAM J. Finance Math. 3, 66-94 (2011)

20. Nicolato, E., Venardos, E.: Option pricing in stochastic volatility models of the OrnsteinUhlenbeck type. Math. Finance 13, 445-466 (2003)

21. Pigorsch, C., Stelzer, R.: A multivariate Ornstein-Uhlenbeck type stochastic volatility model. Working paper (2009) http://www.uni-ulm.de/mawi/finmath/people/stelzer/publications.html

22. Protter, P.: Stochastic Integration and Differential Equations. Stochastic Modelling and Applied Probability, vol. 21, 2nd edn. Springer, New York (2004)

23. Raible, S.: Lévy Processes in Finance: Theory, Numerics and Empirical Facts. Dissertation, Mathematische Fakultät, Albert-Ludwigs-Universität Freiburg i. Br., Freiburg, Germany, (2000)

24. Sato, K.: Lévy Processes and Infinitely Divisible Distributions, volume 68 of Cambridge Studies in Advanced Mathematics. Cambridge University Press, Cambridge (1999) 
25. Schoutens, W.: Lévy Processes in Finance-Pricing Financial Derivatives. Wiley, Chicester (2003)

26. Stelzer, R, Tosstorff, T, Wittlinger, M.: Moment based estimation of supOU processes and a related stochastic volatility model. submitted for publication (2013) http://arxiv.org/abs/1305. $1470 \mathrm{v} 1$

27. Veretennikov, A.Y.: On lower bounds for mixing coefficients of Markov diffusions. In: Kabanov, Y., Lipster, R., Stoyanov, J. (eds.) From Stochastic Calculus to Mathematical Finance-The Shiryaev Festschrift, pp. 33-68. Springer, Berlin (2006) 